\title{
Pilot Scheme of Health Policy in Stroke Adjuvant Acupuncture Therapy for Acute and Subacute Ischemic Stroke in Taiwan
}

\author{
Yi-Chia Wei, ${ }^{1}$ Mao-Feng Sun, ${ }^{2,3}$ Ku-Chou Chang, ${ }^{4,5,6,7}$ Chee-Jen Chang, ${ }^{8,9}$ \\ Yu-Chiang Hung, ${ }^{6,10}$ Yu-Jr Lin,, ${ }^{11}$ and Hsien-Hsueh Elley Chiu ${ }^{6,12}$ \\ ${ }^{1}$ Department of Neurology, Chang Gung Memorial Hospital at Keelung, Keelung 20401, Taiwan \\ ${ }^{2}$ Department of Acupuncture, China Medical University Hospital, Taichung 40447, Taiwan \\ ${ }^{3}$ School of Chinese Medicine, China Medical University, Taichung 40402, Taiwan \\ ${ }^{4}$ Department of Neurology, Division of Cerebrovascular Diseases, \\ Chang Gung Memorial Hospital_Kaohsiung Medical Center, Kaohsiung 83301, Taiwan \\ ${ }^{5}$ Center of Discharge Planning Service, Chang Gung Memorial Hospital_Kaohsiung Medical Center, Kaohsiung 83301, Taiwan \\ ${ }^{6}$ College of Medicine, Chang Gung University, Kaohsiung 83301, Taiwan \\ ${ }^{7}$ Yuh-Ing Junior College of Health Care and Management, Kaohsiung 80776, Taiwan \\ ${ }^{8}$ Graduate Institute of Clinical Medical Sciences, Chang Gung University, Taoyuan 33302, Taiwan \\ ${ }^{9}$ Clinical Informatics and Medical Statistics Research Center, Chang Gung University, Taoyuan 33302, Taiwan \\ ${ }^{10}$ Department of Traditional Chinese Medicine, Division of Internal Medicine, \\ Chang Gung Memorial Hospital_Kaohsiung Medical Center, Kaohsiung 83301, Taiwan \\ ${ }^{11}$ Resource Center for Clinical Research, Chang Gung Memorial Hospital at Linkou, Taoyuan 33305, Taiwan \\ ${ }^{12}$ Department of Traditional Chinese Medicine, Division of Acupuncture and Chinese Traumatology, \\ Chang Gung Memorial Hospital_Kaohsiung Medical Center, Kaohsiung 83301, Taiwan
}

Correspondence should be addressed to Hsien-Hsueh Elley Chiu, elley@adm.cgmh.org.tw

Received 10 August 2010; Revised 28 December 2010; Accepted 7 February 2011

Copyright (c) 2011 Yi-Chia Wei et al. This is an open access article distributed under the Creative Commons Attribution License, which permits unrestricted use, distribution, and reproduction in any medium, provided the original work is properly cited.

\begin{abstract}
To reduce the health care burden of strokes, the Taiwan Department of Health launched the Pilot Scheme of the Health Policy in Stroke Adjuvant Acupuncture Therapy (HPSAAT) in 2006. This cross-sectional, hospital-based, match-controlled study at Chang Gung Memorial Hospital-Kaohsiung Medical Center during 2006 2008 retrospectively evaluated the clinical characteristics of acute and subacute ischemic stroke patients who electively joined the HPSAAT. The study also evaluated the safety and clinical benefits of adjuvant acupuncture in treating acute and subacute ischemic stroke patients. Twenty-six HPSAAT participants and 52 age-sex matched random controls were enrolled. The stroke baseline of the HPSAAT participants was more severe than the nonHPSAAT controls. Although the stroke severity closely correlates to mortality and comorbidity, this study noted no significant complications in the HPSAAT participants during the acupuncture treatment course. Adjuvant acupuncture was considered safe at the acute and subacute stages of ischemic stroke. Due to uneven baseline severity, the clinical benefits in reducing neurological deficits and functional recovery were not concluded in this study.
\end{abstract}

\section{Introduction}

As one of the leading causes of death, strokes contribute to a worldwide health care burden $[1,2]$. Acupuncture has long been applied in treating stroke patients and has thus been recommended by the World Health Organization [3]. The National Health Insurance (NHI) is the social insurance that has covered the medical expenses of over $99 \%$ of 23.0 million Taiwanese citizens since 1995 [4-6]. In Taiwan, acupuncture prevailed in one fifth of NHI beneficiaries [7]. Aiming to improve the health care of stroke patients, the Department of Health launched an NHI-sponsored national project in 2006, namely the Pilot Scheme of the Health Policy in Stroke Adjuvant Acupuncture Therapy (HPSAAT). The HPSAAT promotes integration of traditional Chinese medicine (TCM) in conventional stroke care. In 2010, the HPSAAT has been conducting in 27 medical centers and local hospitals under an annual budget of 2.7 million US dollars. 
Patients with newly onset stroke (during the past year) receive support and elective acupuncture. Both inpatient treatment and outpatient clinic visits are covered.

Along the clinical course of ischemic stroke, the most important period of recovery is at the acute and subacute stage [8]. In this study, we sought to identify the characteristics of acute and subacute stroke patients who electively chose to participate in the HPSAAT. We also aimed to evaluate the safety of adjuvant acupuncture in stroke inpatient care.

Among patients with stroke, the major cause of death is from medical complications [9] that mostly develop within the first 6 weeks after stroke onset [10]. The incident rate of medical complications increases with the baseline severity of stroke. A higher score of the National Institution of Health Stroke Scale (NIHSS) [11, 12] or a higher dependency at stroke onset [10] is correlated to an increase of medical complications. Among all medical complications during acute and subacute stroke, infection is the most common medical complication [13] and is an independent predictor of mortality and poor functional outcome $[9,14,15]$. The high prevalence of poststroke infections, as well as other postCNS (central nervous system) injury infections, has been supposed to be the consequence of CNS immunodepression with imbalanced subsets of helper $T$ cells $[16,17]$. In acupuncture theory, correcting imbalances is the ultimate goal of needling. Both proinflammatory and inflammatory effects have been observed in acupuncture studies [18]. The postacupuncture sequential change of peripheral blood leukocyte subpopulation and cytokines reveals immuneboosting effects $[19,20]$. Based on the previous findings, we hypothesize that acupuncture has the potential to correct CNS injury-induced immunodepression and reduce the infectious complications of stroke patients. In this study, we attempted to use age-sex matched control to evaluate the clinical benefits of adjuvant acupuncture in reducing medical complications in HPSAAT participants.

The other major burden in stroke care involves neurological deficits and functional impairment. The acute and subacute stages account for the golden time of stroke recovery [8]. The plasticity of the brain significantly affects stroke recovery [21]. In recent systematic reviews of acupuncture in acute ischemic stroke, potential therapeutic effects have been noticed [22, 23]. Acupuncture increases regional cerebral blood flow in the hypoperfusion area surrounding the ischemic core and in the sensorimotor area of the affected and unaffected hemisphere of the brain in ischemic stroke patients [24]. A functional MRI study confirmed that acupuncture elicits higher signals in the somatosensory area of poststroke brains [25]. In this study, we compared the neurological improvement between the HPSAAT participants and age-sex matched controls.

\section{Method}

2.1. Study Design and Patients. This research involved a cross-sectional, hospital-based, matched-control study conducted at Chang Gung Memorial Hospital-Kaohsiung Medical Center (KCGMH). The study was approved by the institutional review board of KCGMH. Acute and subacute stroke patients admitted to the neurology ward and neurology intensive care unit (NICU) from 1 July 2006 to 30 June 2008 were assessed for eligibility. Ischemic stroke is defined by ICD-9 code 434 . Only admissions for initial management of acute and subacute ischemic stroke were included. Readmissions of a single patient during the investigation period were excluded. Among ischemic stroke patients, some patients electively joined the HPSAAT to receive adjuvant acupuncture. Occasionally, families of patients with cognitive impairment made the decision regarding HPSAAT participation. After patients began participating in the HPSAAT, TCM doctors received a consultation sheet, visited the HPSAAT participants, and began the treatment course.

In this study, HPSAAT participants were allocated to case groups. Matched control group members were randomly selected among the nonHPSAAT participants by $1: 2$ agesex matching [26]. Those cases whose medical records did not present sufficient neurological descriptions for NIHSS scoring were excluded. The enrolled cases were retrieved for full chart review of the admission course and 6-month period of poststroke followup at KCGMH (Figure 1).

2.2. Procedures. All patients received concomitant stroke care in the neurology ward and/or in NICU. In addition, experienced TCM doctors visited the HPSAAT participants three times per week and performed acupuncture treatment. The acupuncture point selection was determined by doctor clinical evaluation in accordance with TCM principles. After manual acupuncture, the disposable stainless needles were retained for 15 minutes. When clinical conditions deteriorated or infectious conditions occurred, doctors of both neurology and TCM could evaluate the patients and decide if acupuncture should be administered.

2.3. Data Collection. The demographic profiles recorded age, sex, and body mass index. The clinical profile regarding strokes recorded stroke types, lesion sites, previous strokes, and risk factors. The population profile recorded patient sources, dispositions, and timescale of treatment. The medical resource profile recorded thrombolytic therapy (r-tPA), NICU stay, craniectomy or shunt implantation, mechanical ventilation, bedside physical therapy, and length of stay. Confounding factors of outcome were assessed by a baseline NIHSS [27] and a baseline modified Rankin Scale (mRS) [28]. We retrospectively scored the NIHSS $[29,30]$ and mRS by an independent clinical investigator certified by the American Heart Association professional education center [31].

Neurological complications were recorded as recurrent stroke during admission and stroke-in-evolution [32]. Medical complications during admission were evaluated by urinary tract infection, pneumonia, and cellulitis [13]. Also the stress events represented by gastrointestinal (GI) bleeding episodes, hemodynamic stability evaluated by vital sign at 09:00 a.m. of the day next to admission day and of the day before discharge day. Significant adverse events of 


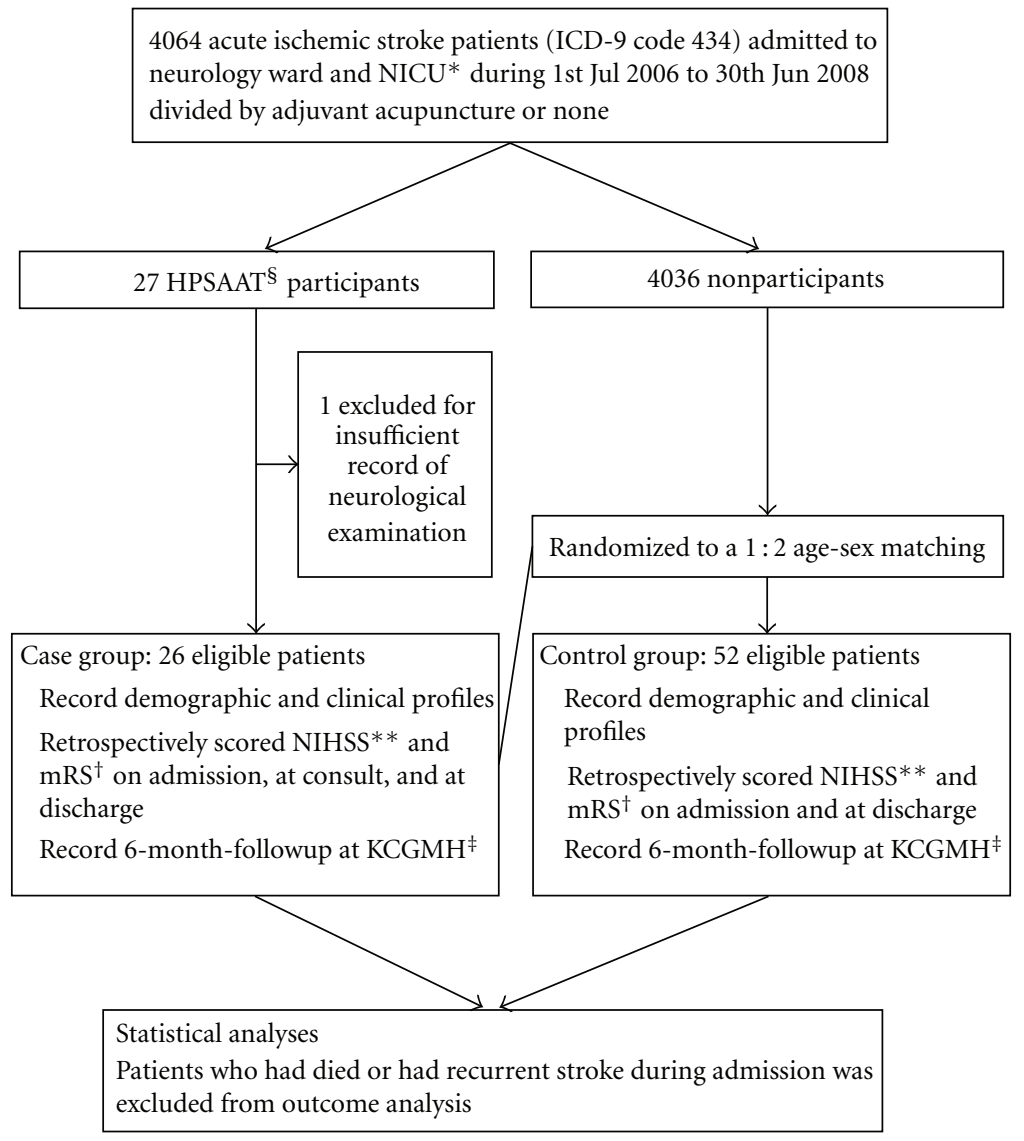

FIGURE 1: Participant flowsheet. *NICU, neurology intensive care unit. ${ }^{* *}$ NIHSS, National Institutes of Health Stroke Scale. Scored by one independent physician with certification of American Heart Association Professional Education Center website. ${ }^{\dagger}$ mRS, modified Rankin Scale. ${ }^{\ddagger}$ KCGMH, the Chang Gung Memorial Hospital-Kaohsiung Medical Center. ${ }^{\S}$ HPSAAT, the Pilot Scheme of the Health Policy in Stroke Adjuvant Acupuncture Therapy. HPSAAT provides stroke patients elective acupuncture at outpatients and inpatients departments during the first year after stroke onset.

acupuncture were reviewed [33]. We also traced patient admission records and outpatient visits at KCGMH for a 6-month period. Patients who had died or had a recurrent stroke during admission were separated from outcome analysis.

2.4. Statistical Analysis. Categorical data were expressed by frequency (percentage) and were examined by a chi-squared test. Continuous data were expressed by mean (standard deviation) and were examined by an independent $t$-test. A $P$ value $<.05$ in a 2 -tailed test was considered to be statistically significant. Data were analyzed using a commercial software program, SPSS version 17.0 for Windows; SPSS Inc.

\section{Results}

This study involved 273 HPSAAT participants, most of them were in the stroke recovery stage and admitted in rehabilitation wards. Only 27 participants with acute and subacute ischemic stroke were eligible for the case group. One case was further excluded due to insufficient neurological examination records for retrospective NIHSS scoring, leaving 26 participants in the case group. Fiftytwo age-sex matched controls were randomly selected from 4,036 acute and subacute ischemic stroke patients who did not participate in the HPSAAT but received conventional treatment indiscriminately (Figure 1).

The characteristic profile of the HPSAAT participants and age-sex matched nonHPSAAT controls showed no difference in demographics, stroke type (Table 1(a)), or patient source (Table 1(b)). However, the HPSAAT participants seemed to have longer treatment courses, including length of stay in NICU, in the neurology ward, and in total $(32.9 \pm 21.9: 13.8 \pm 16.1$ days, $P<.001)$. Compared to the nonHPSAAT controls, the HPSAAT participants tended to stay in health care institutions after discharge from the neurology ward and/or NICU, including the rehabilitation ward, TCM ward, and long-term care units (Table 1(b)). Bedside physical therapy was more prevalently applied to the HPSAAT participants (92.3\%:53.8\%, $P=.001$; Table $1(\mathrm{c})$ ).

The HPSAAT participants in the neurology ward and NICU started adjuvant acupuncture an average of $17.7 \pm 14.4$ days after stroke onset and continued the treatment course for $17.4 \pm 16.2$ days with $6.7 \pm 6.4$ acupuncture sessions (Table 1(b)). The safety of acupuncture was evaluated in a 
TABLE 1: Clinical characteristics.

\begin{tabular}{|c|c|c|c|}
\hline & $\begin{array}{l}\text { HPSAAT participants } \\
(n=26)\end{array}$ & $\begin{array}{l}\text { Matched control: non } \\
\text { HPSAAT }(n=52)\end{array}$ & $P$-value \\
\hline \multicolumn{4}{|c|}{ (a) Demographics } \\
\hline Age, years* & $70.2(11.3)$ & $70.2(11.1)$ & Matched \\
\hline Sex, male** & $16(61.5)$ & $32(61.5)$ & Matched \\
\hline Body mass index, $\mathrm{kg} / \mathrm{m}^{2 *}$ & $23.5(3.2)$ & $23.8(3.6)$ & \\
\hline Stroke ever** & $14(53.8)$ & $26(50.0)$ & \\
\hline \multicolumn{4}{|l|}{ Stroke type** } \\
\hline Ischemic & $23(88.5)$ & $50(96.2)$ & \\
\hline $\begin{array}{l}\text { Ischemic with hemorrhagic } \\
\text { transformation }\end{array}$ & $3(11.1)$ & $2(3.7)$ & \\
\hline \multicolumn{4}{|l|}{ Stroke lesion site ${ }^{* *}$} \\
\hline Right cerebral hemisphere & $5(19.2)$ & $16(30.8)$ & \\
\hline Left cerebral hemisphere & $8(30.8)$ & $15(28.8)$ & \\
\hline Cerebellum & $0(0.0)$ & $2(3.8)$ & \\
\hline Brain stem & $5(19.2)$ & $11(21.2)$ & \\
\hline Multiple infarcts ( $\geq 2$ of above) & $8(30.8)$ & $8(15.4)$ & \\
\hline \multicolumn{4}{|l|}{ Risk factors of stroke** } \\
\hline Hypertension & $20(76.9)$ & $33(63.5)$ & \\
\hline Diabetes mellitus & $14(53.8)$ & $25(48.1)$ & \\
\hline Hypertriglyceridemia & $7(26.9)$ & $10(19.2)$ & \\
\hline Hypercholesterolemia & $11(42.3)$ & $17(32.7)$ & \\
\hline Atrial fibrillation & $5(19.2)$ & $6(11.5)$ & \\
\hline Coronary artery disease & $5(19.2)$ & $4(7.7)$ & \\
\hline Congestive heart failure & $0(0.0)$ & $1(1.9)$ & \\
\hline Cigarette smoking & $6(23.1)$ & $16(30.8)$ & \\
\hline Obesity & $7(31.8)$ & $14(33.3)$ & \\
\hline
\end{tabular}

(b) Patients' source, treatment course, and disposition

Patient source**

Emergency room

Referred from other hospital

Outpatients

Referred from other ward in

hospital

Timescale of treatment, days*

Onset to ward

$$
\begin{gathered}
20(76.9) \\
3(11.5) \\
1(3.8) \\
2(7.7) \\
\\
2.9(4.7) \\
17.7(14.4) \\
17.4(16.2) \\
6.7(6.4) \\
2.9(1.5) \\
11.6(14.1) \\
19.2(8.6) \\
2.0(5.5) \\
32.9(21.9)
\end{gathered}
$$

Length of stay, days ${ }^{*, \neq}$

In NICU

In neurology ward

\section{Disposition**,‡}

Home

$16(64.0)$

Rehabilitation ward

Traditional Chinese medicine ward

$4(16.0)$

$1(4.0)$

$5(20.0)$

Long-term care unit ${ }^{\S}$
39 (75.0)

$4(7.7)$

9 (17.3)

$0(0.0)$

$2.3(3.1)$

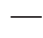

-

-

$\begin{array}{cc}2.2(4.7) & <.01 \\ 11.6(12.7) & <.01 \\ 0.0(0.0) & <.001 \\ 13.8(16.1) & \\ 46(95.8) & .001 \\ 0(0.0) & <.05 \\ 0(0.0) & \\ 2(4.2) & <.05\end{array}$


TABle 1: Continued.

\begin{tabular}{lcc}
\hline & $\begin{array}{c}\text { HPSAAT participants } \\
(n=26)\end{array}$ & $\begin{array}{c}\text { Matched control: non } \\
\text { HPSAAT }(n=52)\end{array}$ \\
\hline Received r-tPA & (c) Medical resource use** & \\
Ever NICU stay & $0(0.0)$ & $0(0.0)$ \\
Craniectomy or shunt implantation & $17(65.4)$ & $15(28.8)$ \\
Mechanical ventilation & $2(7.7)$ & $0(0.0)$ \\
Received bedside physical therapy & $4(15.4)$ & $2(3.8)$ \\
\hline
\end{tabular}

${ }^{*}$ Data are given as mean (SD). ${ }^{* *}$ Data are given as number (percentage). ${ }^{\dagger}$ Mortality was excluded (case $=0$, control $\left.=3\right) .{ }^{\ddagger}$ Mortality $($ case $=0$, control $=3$ ) and patients with recurrent stroke during admission ( case $=1$, control $=1$ ) were excluded. ${ }^{\S}$ Long-term care unit included local hospital and nursing home. Abbreviation: HPSAAT: the Pilot Scheme of the Health Policy in Stroke Adjuvant Acupuncture Therapy. NICU: neurology intensive care unit. RCC: respiratory care center. r-tPA: thrombolytic therapy.

detailed chart review. No vital sign instability was noted among the HPSAAT participants during acupuncture. The baseline vital sign on admission was not significantly different between groups; whereas, the HPSAAT participants presented lower diastolic blood pressure (76.8 \pm 8.5:82.1 \pm $9.7 \mathrm{mmHg}, P<.05)$, lower mean arterial pressure $(95.6 \pm$ $8.9: 100.7 \pm 10.7 \mathrm{mmHg}, P<.05)$, and faster heart rate $(79.1 \pm 8.1: 73.7 \pm 7.2$ beat per minute, $P<.01)$ than the nonHPSAAT controls. The adverse event survey revealed no needle sickness. In one participant, left-arm cellulitis developed 8 days after acupuncture initiation (cause undetermined). An intravenous antibiotic was administered and the acupuncture treatment was uninterrupted and completed until the patient was discharged home. Regarding another participant who had begun acupuncture treatment in the neurology ward, the acupuncture treatment was interrupted after two sessions (7 days) of treatment because of sudden collapse and admission to NICU. The other 24 HPSAAT participants completed the acupuncture treatment course smoothly during the stay in the neurology ward and/or NICU.

The mortality and comorbidity analysis began from a baseline comparison. The baseline NIHSS was higher on average $(14.9 \pm 9.4: 7.5 \pm 7.0, P<.001$; Table $2(\mathrm{a}))$ and was skewed more to the left in distribution (Figure 2(a)) among the HPSAAT participants than that of the nonHPSAAT controls. The baseline mRS of the HPSAAT participants was also more severe than that of the nonHPSAAT controls (dependent as $\mathrm{mRS}>3$ in $92.3 \%: 61.5 \%, P<.01$ ), but was distributed similarly (Figure 2(b)). Three enrolled cases expired during admission. All mortalities occurred in the control group and were caused by severe infection with septic shock. A comparison of neurological and medical complications discovered more stroke-in-evolution $(34.6 \%: 3.8 \%$, $P=.001)$ and urinary tract infection $(46.2 \%: 23.1 \%, P<$ $.05)$ in the HPSAAT participants than in the nonHPSAAT controls during stays in the neurology ward and/or NICU. However, more urinary tract infections occurred before initiating acupuncture (Table 2(b)). No difference was noted in the occurrences of recurrent stroke, pneumonia, cellulitis, or GI bleeding.

In the 6-month followup period, the HPSAAT participants tended to maintain regular outpatient visits to the rehabilitation department $(20.0 \%: 2.1 \%, P<.05)$ and acupuncture department $(32.0 \%: 0.0 \%, P<.001)$. Approximately seventy percent of cases in both groups continued following up in the neurology department for at least 6 months after stroke onset.

\section{Discussion}

In this study, the major finding, but also the major limitation, was the uneven baseline severity between the HPSAAT participants and the age-sex matched nonHPSAAT controls. The acute and subacute ischemic stroke patient with severe neurological impairment and significantly functional limitations was prone to join the HPSAAT for adjuvant acupuncture in addition to conventional treatment. This finding indicated the desire of major ischemic stroke patients and their families to pursue satisfying treatment modalities or a combination of treatment modalities. This finding also indicated that the developmental principle of acupuncture in treating ischemic stroke should be emphasized on moderate to severe patients.

However, the baseline discrepancy limited the effective comparison of neurological and functional outcomes. The alternative solution may come from data mining in the Chang Gung Stroke Registry Database, which includes clinical data of all stroke patients admitted to the four branches of Chang Gung Memorial Hospital since 2008. Searching by the NIHSS on admission could find the baseline equaled populations in the database.

The varied duration of stroke onset to acupuncture treatment $(17.7 \pm 14.4$ days $)$ and varied duration of acupuncture treatment $(17.4 \pm 16.2$ days $)$ among the HPSAAT participants also limited the comparison of benefits from adjuvant acupuncture in ischemic stroke. For more accurate generalization and more effective comparison, a randomized control trial is required.

Since the 1990s, the efficacy and effectiveness of acupuncture for acute and subacute stroke has been evaluated by many research groups. Although some study designs have been limited by the strength of evidence, systemic reviews revealed the potential benefits of acupuncture to patients with acute and subacute strokes $[22,23]$. The HPSAAT is the first public health policy-modifying project to include 
TABLE 2: Mortality and comorbidity during admission and in six-month-followup.

\begin{tabular}{|c|c|c|c|}
\hline & $\begin{array}{c}\text { HPSAAT participants } \\
(n=26)\end{array}$ & $\begin{array}{c}\text { Matched control: non } \\
\text { HPSAAT }(n=52)\end{array}$ & $P$-value \\
\hline \multicolumn{4}{|c|}{ (a) Baseline comparison: factors affecting mortality and comorbidity } \\
\hline \multicolumn{4}{|l|}{ Neurological impairment* } \\
\hline Baseline NIHSS scores & $14.9(9.4)$ & $7.5(7.0)$ & $<.001$ \\
\hline Interquartile range & $7-22$ & $2-11.75$ & \\
\hline NIHSS at consult & $16.6(8.8)$ & - & \\
\hline NIHSS at discharge ${ }^{\ddagger}$ & $15.4(8.3)$ & $5.6(5.5)$ & $<.001$ \\
\hline \multicolumn{4}{|l|}{ Functional impairment** } \\
\hline Baseline $\mathrm{mRS}>3$ & $24(92.3)$ & $32(61.5)$ & $<.01$ \\
\hline $\mathrm{mRS}>3$ at consult & $25(96.2)$ & - & \\
\hline $\mathrm{mRS}>3$ at discharge $\mathrm{e}^{\ddagger}$ & $22(88.0)$ & $24(50.0)$ & .001 \\
\hline \multicolumn{4}{|c|}{ (b) Mortality and comorbidity during admission ${ }^{* *}$} \\
\hline Mortality during admission & $0(0.0)$ & $3(5.8)$ & \\
\hline \multicolumn{4}{|l|}{ Neurological complications } \\
\hline Recurrent stroke & $1(3.8)$ & $1(1.9)$ & \\
\hline Stroke in-evolution after admission & $9(34.6)$ & $2(3.8)$ & .001 \\
\hline \multicolumn{4}{|l|}{ Medical complications } \\
\hline Urinary tract infection (total) & $12(46.2)$ & $12(23.1)$ & $<.05$ \\
\hline Before acupuncture & $7(26.9)$ & - & \\
\hline After acupuncture & $5(19.2)$ & - & \\
\hline Pneumonia (total) & $9(34.6)$ & $11(21.2)$ & \\
\hline Before acupuncture & $9(34.6)$ & - & \\
\hline After acupuncture & $0(0.0)$ & - & \\
\hline Cellulitis (total) & $2(7.7)$ & $1(1.9)$ & \\
\hline Before acupuncture & $1(3.8)$ & - & \\
\hline After acupuncture & $1(3.8)$ & - & \\
\hline Gastrointestinal bleeding & $4(15.4)$ & $8(15.4)$ & \\
\hline Before acupuncture & $4(15.4)$ & - & \\
\hline After acupuncture & $0(0.0)$ & - & \\
\hline
\end{tabular}

(c) Six-month followup in $\mathrm{KCGMH}^{* *, \neq}$

Ever readmission due to acute disorders

Recurrent stroke within 6 months

Expire within 6 months

Outpatient followup ${ }^{* *, \neq}$

In neurology department

$\begin{array}{lll}\text { First } 3 \text { months } & 19(76.0) & 37(77.1) \\ 6 \text { months } & 17(68.0) & 37(77.1)\end{array}$

In rehabilitation department

First 3 months $\quad 5(20.0)$

6 months $\quad 5(20.0)$

In acupuncture department

First 3 months $9(36.0)$

6 months

$\begin{array}{ll}2(8.0) & 7(14.6) \\ 0(0.0) & 1(2.1) \\ 0(0.0) & 2(4.2)\end{array}$

\footnotetext{
${ }^{*}$ Data are given as mean $(\mathrm{SD}) .{ }^{* *}$ Data are given as number (percentage). ${ }^{\dagger}$ Mortality was excluded $($ case $=0$, control $=3) .{ }^{\ddagger}$ Mortality $($ case $=0$, control $=3$ ) and patients with recurrent stroke (case $=1$, control $=1$ ) during admission were excluded. Abbreviation: HPSAAT, the Pilot Scheme of the Health Policy in Stroke Adjuvant Acupuncture Therapy. NIHSS: National Institutes of Health Stroke Scale. mRS: modified Rankin Scale. mRS > 3 was defined as dependent status. KCGMH: the Chang Gung Memorial Hospital—Kaohsiung Medical Center.
} 

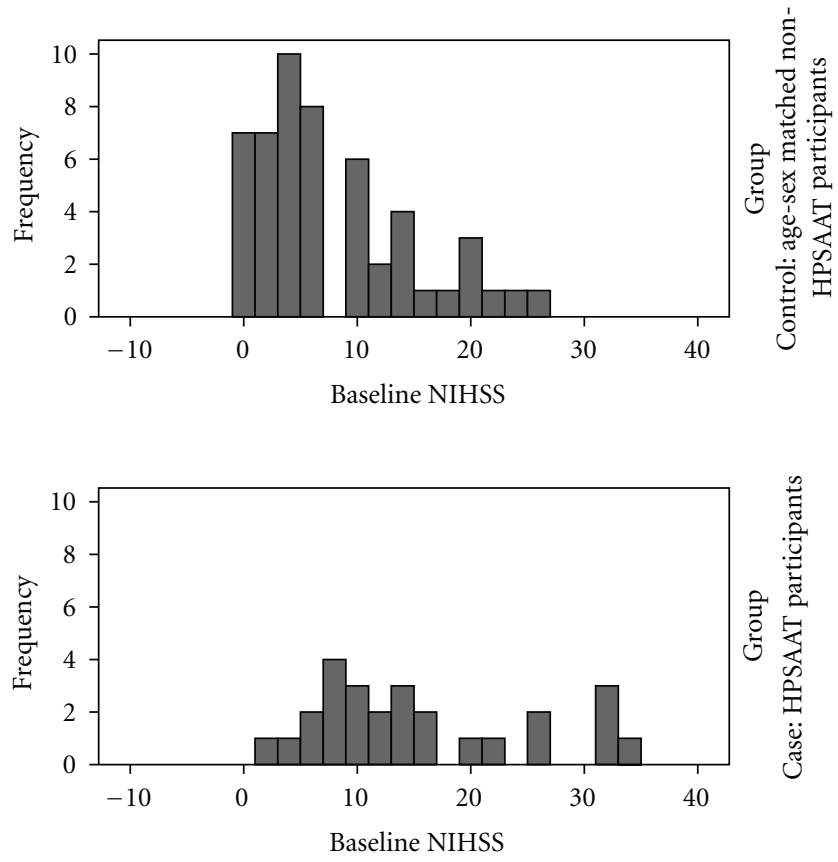

(a) Histogram of Baseline NIHSS

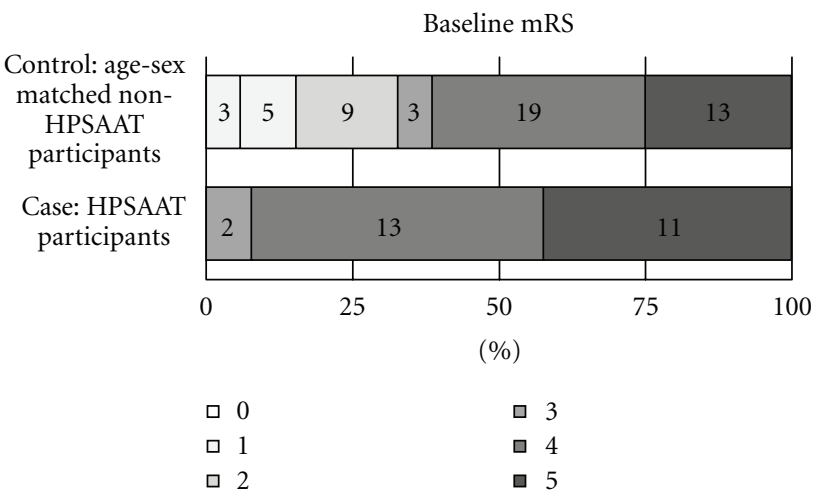

(b) Baseline mRS (case numbers and percentages)

FIGURE 2: Baseline discrepancies between HPSAAT participants and age-sex matched nonparticipants. The histogram of baseline NIHSS showed the different distribution of severity between groups. There were more moderate to severe strokes among the HPSAAT participants with acute and subacute ischemic stroke than the agesex matched nonHPSAAT controls (a). The number and percentage of baseline mRS showed that the HPSAAT participants were more dependent than the random controls (b).

acupuncture in stroke care. Previous evidence has suggested that the worse the baseline is, the worse the outcomes are. Moderate to severe strokes had poorer outcomes [34, 35], greater demand of long-term care [36, 37], and higher expense of medical costs [38]. The acute and subacute stages constitute the golden recovery period for ischemic stroke. From this study, we found that major ischemic stroke patients tended to choose adjuvant acupuncture in the acute and subacute stages. Future studies must clarify whether or not the HPSAAT reduced the financial burden. Moreover, the baseline severity also correlated to the increased poststroke infections [12]. In this study, a higher rate of urinary tract infection of the HPSAAT participants, but not of pneumonia or GI bleeding, was noted. The infections that occurred after starting acupuncture treatment did not seem significantly increased. Therefore, in addition to improving neurological impairment or functional recovery, acupuncture may play a role in eliminating medical complications of stroke. The immune-boosting potentials of acupuncture in modulating CNS injury-induced immunodepression of acute and subacute stroke is still worthy of further investigation.

The 6-month followup period revealed favorable compliance of the HPSAAT participants to continue acupuncture treatment after discharge. The needs of long-term functional recovery and the selection of acupuncture as a treatment modality was noticed in one third of the HPSAAT participants.

\section{Conclusion}

This cross-sectional study analyzed data from a single medical center to depict a brief summary of the HPSAAT in Taiwan. Adjuvant acupuncture care is a safe modality in the acute and subacute stages of ischemic stroke. Ischemic stroke patients with a severe baseline tended to participate in the HPSAAT. To validate the clinical benefits of adjuvant acupuncture care in acute and subacute ischemic stroke, a baseline equaled randomized control trial is warranted.

\section{Statement of Contribution}

We state the contribution of Yi-Chia Wei MD in data acquirement, data analysis, and manuscript drafting, MaoFeng Sun $\mathrm{MD} / \mathrm{PhD}$ in HPSAAT project supervision, $\mathrm{Ku}-$ Chou Chang MD in data acquiring and study supervision, Chee-Jen Chang PhD in study design, Yu-Chiang Hong $\mathrm{MD} / \mathrm{PhD}$ in supervision of this study, Yu-Jr Lin MSc in performing statistical analysis, and Hsien-Hsueh Elley Chiu MD in conducting HPSAAT, conceiving and design the research, analyzing and interpreting the data, and making critical revision of the manuscript.

\section{Conflicts of Interest}

All authors and contributors declared that no conflict of interest exists.

\section{Funding}

This study was supported by the Chung Gung Research Project, CMRPG880551. The funding source had no role in the study design, data collection, data analysis, data interpretation, or writing of the report. The corresponding author and the first author had full access to all data in the study and had final responsibility for the decision to submit the paper for publication. 


\section{Acknowledgments}

The authors give thanks to the Taiwan Traditional Chinese Medicine Association for providing information of HPSAAT. We acknowledge the contribution of Hen-Hong Chang $\mathrm{MD} / \mathrm{PhD}$ and Sheng-Teng Huang $\mathrm{MD} / \mathrm{PhD}$ in the administration support of this study, and Jer-Ming Sheen MD, Cheng-Nan Lu MD, Guo-Wei Bi MD, and Wu-Long Hu MD in treating the HPSAAT patients in KCGMH.

\section{References}

[1] WHO, Global Burden of Disease: Statistics, WHO, Geneva, Switzerland, 2004.

[2] Department H. Statistics of Cause of Death 2008. In. Taipei, Taiwan (R.O.C.): Department of Health: 2008.

[3] World Health Organization, Acupuncture: Review and Analysis Reports on Controlled Clinical Trials, WHO, Geneva, Switzerland, 2002.

[4] C. T. Liu, "Health care systems in transition II. Taiwan, Part I. A general overview of the health care system in Taiwan," Journal of Public Health Medicine, vol. 20, no. 1, pp. 5-10, 1998.

[5] Department S. Statistical Yearbook of Interior. In. Taipei: Department of Statistics, Ministry of the Interior, Taiwan (R.O.C.): 2009.

[6] N. H. I. Bureau, The National Insurance Statistics 2008. In. Taipei:Bureau of National Health Insurance: 2008.

[7] F. P. Chen, Y. Y. Kung, T. J. Chen, and S. J. Hwang, "Demographics and patterns of acupuncture use in the Chinese population: the Taiwan experience," Journal of Alternative and Complementary Medicine, vol. 12, no. 4, pp. 379-387, 2006.

[8] H. S. Jorgensen, H. Nakayama, H. O. Raaschou, J. ViveLarsen, M. Stoier, and T. S. Olsen, "Outcome and time course of recovery in stroke. Part II: time course of recovery. The Copenhagen Stroke Study," Archives of Physical Medicine and Rehabilitation, vol. 76, no. 5, pp. 406-412, 1995.

[9] K. C. Johnston, J. Y. Li, P. D. Lyden et al., "Medical and neurological complications of ischemic stroke: experience from the RANTTAS trial," Stroke, vol. 29, no. 2, pp. 447-453, 1998.

[10] P. Langhorne, D. J. Stott, L. Robertson et al., "Medical complications after stroke: a multicenter study," Stroke, vol. 31, no. 6, pp. 1223-1229, 2000.

[11] C. Weimar, M. P. Roth, G. Zillessen et al., "Complications following acute ischemic stroke," European Neurology, vol. 48, no. 3, pp. 133-140, 2002.

[12] K. S. Hong, D. W. Kang, J. S. Koo et al., "Impact of neurological and medical complications on 3-month outcomes in acute ischaemic stroke," European Journal of Neurology, vol. 15, no. 12, pp. 1324-1331, 2008.

[13] S. Kumar, M. H. Selim, and L. R. Caplan, "Medical complications after stroke," The Lancet Neurology, vol. 9, no. 1, pp. 105-118, 2010.

[14] F. H. Vermeij, W. J. M. Scholte Op Reimer, P. De Man et al., "Stroke-associated infection is an independent risk factor for poor outcome after acute ischemic stroke: data from the netherlands stroke survey," Cerebrovascular Diseases, vol. 27, no. 5, pp. 465-471, 2009.

[15] H. C. Emsley and S. J. Hopkins, "Acute ischaemic stroke and infection: recent and emerging concepts," The Lancet Neurology, vol. 7, no. 4, pp. 341-353, 2008.

[16] C. Meisel, J. M. Schwab, K. Prass, A. Meisel, and U. Dirnagl, "Central nervous system injury-induced immune deficiency syndrome," Nature Reviews Neuroscience, vol. 6, no. 10, pp. 775-786, 2005.

[17] A. Chamorro, X. Urra, and A. M. Planas, "Infection after acute ischemic stroke: a manifestation of brain-induced immunodepression," Stroke, vol. 38, no. 3, pp. 1097-1103, 2007.

[18] F. J. Zijlstra, I. Van Den Berg-De Lange, F. J. P. M. Huygen, and J. Klein, "Anti-inflammatory actions of acupuncture," Mediators of Inflammation, vol. 12, no. 2, pp. 59-69, 2003.

[19] N. Yamaguchi, T. Takahashi, M. Sakuma et al., "Acupuncture regulates leukocyte subpopulations in human peripheral blood," Evidence-Based Complementary and Alternative Medicine, vol. 4, no. 4, pp. 447-453, 2007.

[20] H. S. Shiue, Y. S. Lee, C. N. Tsai, YU. M. Hsueh, J. R. Sheu, and H. H. Chang, "DNA microarray analysis of the effect on inflammation in patients treated with acupuncture for allergic rhinitis," Journal of Alternative and Complementary Medicine, vol. 14, no. 6, pp. 689-698, 2008.

[21] T. H. Murphy and D. Corbett, "Plasticity during stroke recovery: from synapse to behaviour," Nature Reviews Neuroscience, vol. 10, no. 12, pp. 861-872, 2009.

[22] S. H. Zhang, M. Liu, K. Asplund, and L. Li, "Acupuncture for acute stroke," Cochrane Database of Systematic Reviews, no. 2, Article ID CD003317, 2005.

[23] T. Zhang, L. Zhang, H. M. Zhang, and Q. Li, "Systematic review of acupuncture therapy for acute ischemic stroke," China Journal of Traditional Chinese Medicine and Pharmacy, vol. 24, pp. 101-104, 2009.

[24] J. D. Lee, J. S. Chon, H. K. Jeong et al., "The cerebrovascular response to traditional acupuncture after stroke," Neuroradiology, vol. 45, no. 11, pp. 780-784, 2003.

[25] G. Li, C. R. Jack, and E. S. Yang, "An fMRI study of somatosensory-implicated acupuncture points in stable somatosensory stroke patients," Journal of Magnetic Resonance Imaging, vol. 24, no. 5, pp. 1018-1024, 2006.

[26] E. Girou, F. Schortgen, C. Delclaux et al., "Association of noninvasive ventilation with nosocomial infections and survival in critically ill patients," Journal of the American Medical Association, vol. 284, no. 18, pp. 2361-2367, 2000.

[27] T. Brott, H. P. Adams, C. P. Olinger et al., "Measurements of acute cerebral infarction: a clinical examination scale," Stroke, vol. 20, no. 7, pp. 864-870, 1989.

[28] J. C. Van Swieten, P. J. Koudstaal, M. C. Visser, H. J. A. Schouten, and J. Van Gijn, "Interobserver agreement for the assessment of handicap in stroke patients," Stroke, vol. 19, no. 5, pp. 604-607, 1988.

[29] L. S. Williams, E. Y. Yilmaz, and A. M. Lopez-Yunez, "Retrospective assessment of initial stroke severity with the NIH stroke scale," Stroke, vol. 31, no. 4, pp. 858-862, 2000.

[30] S. E. Kasner, J. A. Chalela, J. M. Luciano et al., "Reliability and validity of estimating the NIH stroke scale score from medical records," Stroke, vol. 30, no. 8, pp. 1534-1537, 1999.

[31] P. Lyden, R. Raman, L. Liu, M. Emr, M. Warren, and J. Marler, "National institutes of health stroke scale certification is reliable across multiple venues," Stroke, vol. 40, no. 7, pp. 2507-2511, 2009.

[32] V. G. Karepov, A. Y. Gur, I. Bova, B. D. Aronovich, and N. M. Bornstein, "Stroke-in-evolution: infarct-inherent mechanisms versus systemic causes," Cerebrovascular Diseases, vol. 21, no. 1-2, pp. 42-46, 2006.

[33] C. M. Witt, D. Pach, B. Brinkhaus et al., "Safety of acupuncture: results of a prospective observational study with 229,230 patients and introduction of a medical information and 
consent form," Forschende Komplementarmedizin, vol. 16, no. 2, pp. 91-97, 2009.

[34] H. P. Adams, P. H. Davis, E. C. Leira et al., "Baseline NIH Stroke Scale score strongly predicts outcome after stroke: a report of the Trial of Org 10172 in Acute Stroke Treatment (TOAST)," Neurology, vol. 53, no. 1, pp. 126-131, 1999.

[35] M. C. Tseng and KU. C. Chang, "Stroke severity and early recovery after first-ever ischemic stroke: results of a hospitalbased study in Taiwan," Health Policy, vol. 79, no. 1, pp. 73-78, 2006.

[36] D. Schlegel, S. J. Kolb, J. M. Luciano et al., "Utility of the NIH stroke scale as a predictor of hospital disposition," Stroke, vol. 34, no. 1, pp. 134-137, 2003.

[37] T. Rundek, H. Mast, A. Hartmann et al., "Predictors of resource use after acute hospitalization: the Northern Manhattan Stroke Study," Neurology, vol. 55, no. 8, pp. 1180-1187, 2000.

[38] K. U. C. Chang and M. C. Tseng, "Costs of acute care of firstever ischemic stroke in Taiwan," Stroke, vol. 34, no. 11, pp. e219-e221, 2003. 


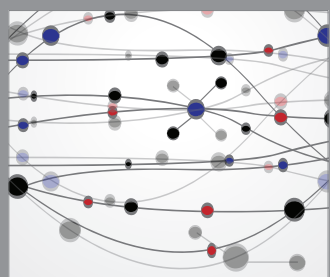

The Scientific World Journal
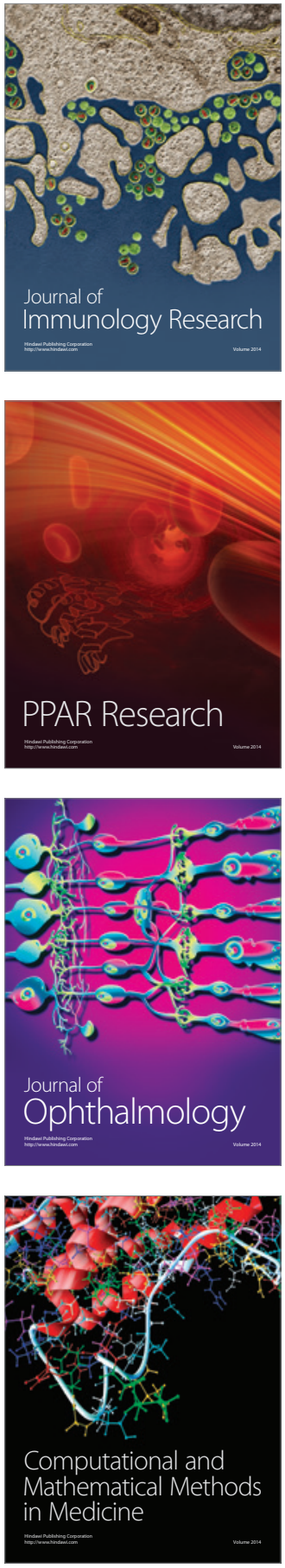

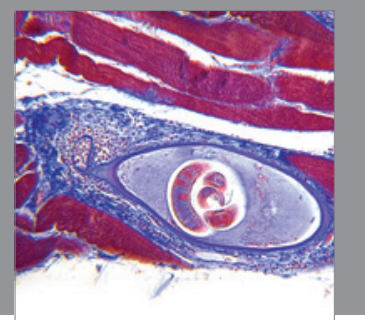

Gastroenterology

Research and Practice
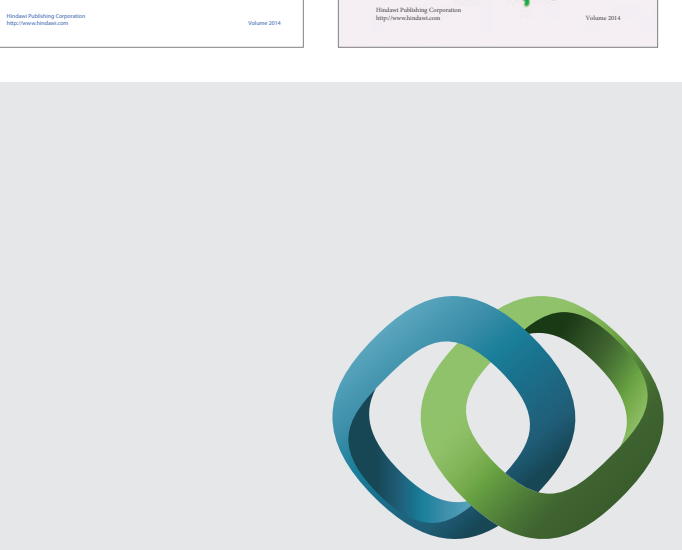

\section{Hindawi}

Submit your manuscripts at

http://www.hindawi.com
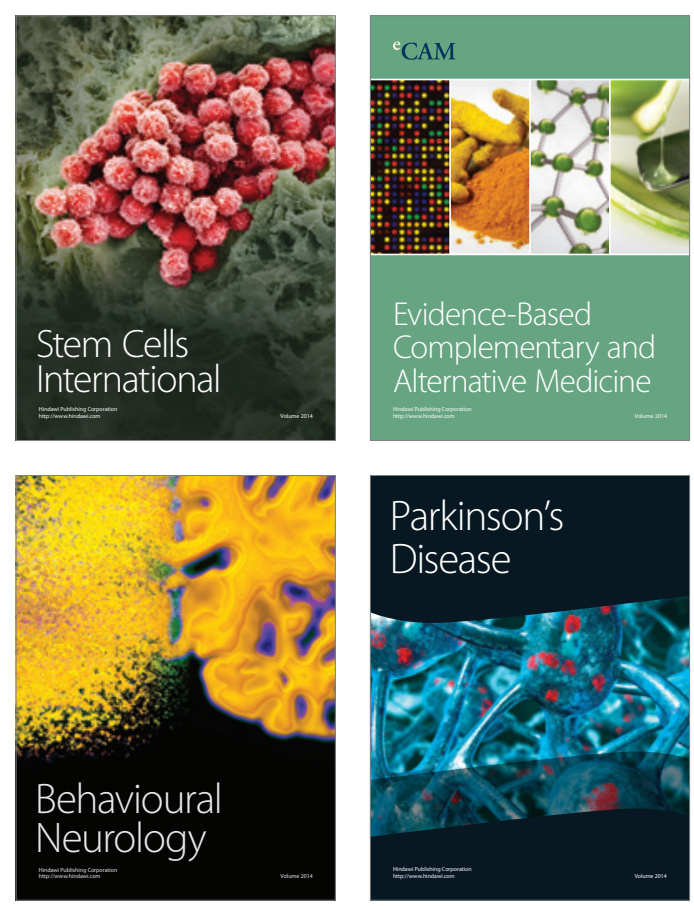

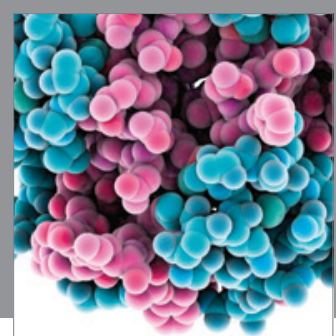

Journal of
Diabetes Research

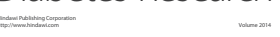

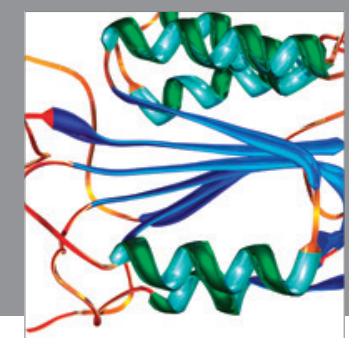

Disease Markers
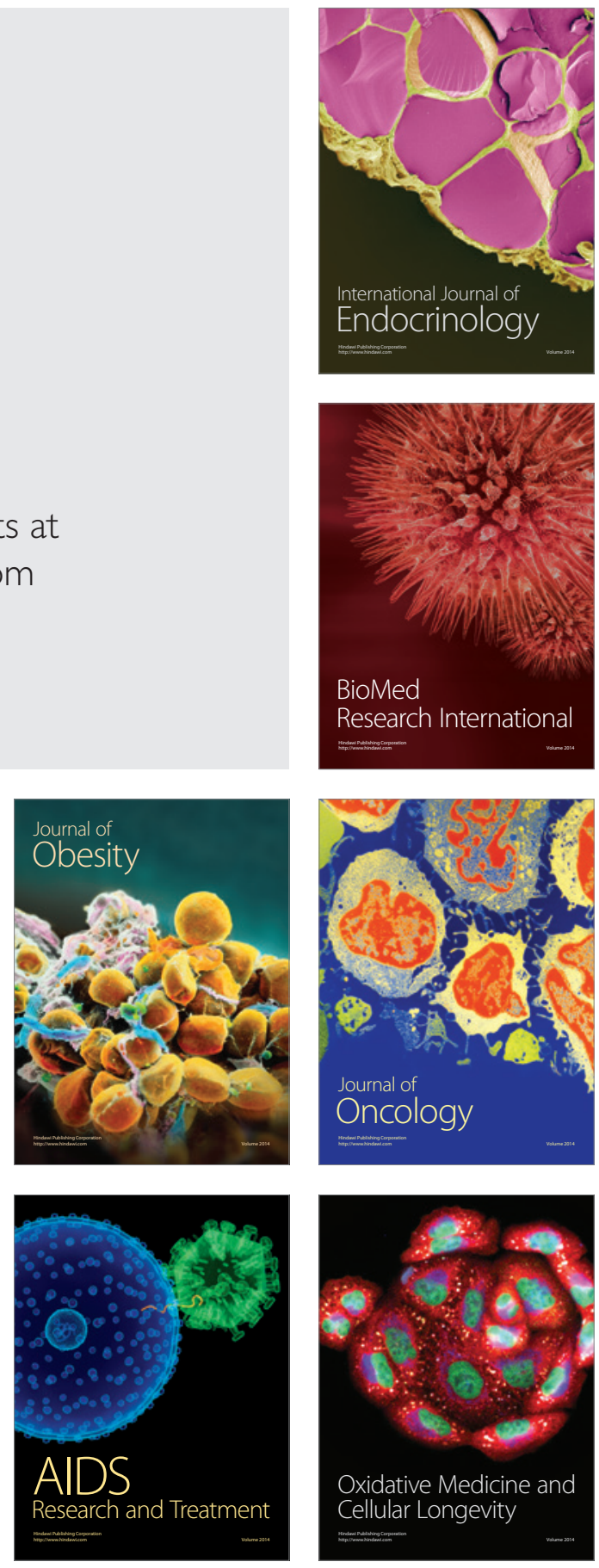